\title{
Clustered microRNAs' coordination in regulating protein-protein interaction network Xiongying Yuan ${ }^{\dagger 1,2}$, Changning Liu ${ }^{\dagger 1}$, Pengcheng Yang ${ }^{1,2}$, Shunmin $\mathrm{He}^{1,2}$, Qi Liao ${ }^{1,3}$, Shuli Kang1 and Yi Zhao*1
}

Address: ${ }^{1}$ Bioinformatics Group, Center for Advanced Computing Research, Institute of Computing Technology, Chinese Academy of Sciences, Beijing, PR China, ${ }^{2}$ Graduate School of Chinese Academy of Sciences, Beijing, PR China and ${ }^{3}$ Department of Parasitology, Zhongshan School of Medicine, Sun Yat-sen University, Guangzhou, PR China

Email: Xiongying Yuan - pkuying@126.com; Changning Liu - lcn@ict.ac.cn; Pengcheng Yang - pengchy@yahoo.com.cn; Shunmin He - heshunmin@gmail.com; Qi Liao - liaoqi04@tom.com; Shuli Kang - kangshuli@gmail.com; Yi Zhao* - biozy@ict.ac.cn

* Corresponding author †Equal contributors

Published: 26 June 2009

BMC Systems Biology 2009, 3:65 doi:10.1 186/1752-0509-3-65
Received: 12 November 2008

Accepted: 26 June 2009

This article is available from: http://www.biomedcentral.com/1752-0509/3/65

(C) 2009 Yuan et al; licensee BioMed Central Ltd.

This is an Open Access article distributed under the terms of the Creative Commons Attribution License (http://creativecommons.org/licenses/by/2.0), which permits unrestricted use, distribution, and reproduction in any medium, provided the original work is properly cited.

\begin{abstract}
Background: MicroRNAs (miRNAs), a growing class of small RNAs with crucial regulatory roles at the post-transcriptional level, are usually found to be clustered on chromosomes. However, with the exception of a few individual cases, so far little is known about the functional consequence of this conserved clustering of miRNA loci. In animal genomes such clusters often contain nonhomologous miRNA genes. One hypothesis to explain this heterogeneity suggests that clustered miRNAs are functionally related by virtue of co-targeting downstream pathways.

Results: Integrating of miRNA cluster information with protein protein interaction (PPI) network data, our research supports the hypothesis of the functional coordination of clustered miRNAs and links it to the topological features of miRNAs' targets in PPI network. Specifically, our results demonstrate that clustered miRNAs jointly regulate proteins in close proximity of the PPI network. The possibility that two proteins yield to this coordinated regulation is negatively correlated with their distance in PPI network. Guided by the knowledge of this preference, we found several network communities enriched with target genes of miRNA clusters. In addition, our results demonstrate that the variance of this propensity can also partly be explained by protein's connectivity and miRNA's conservation.
\end{abstract}

Conclusion: In summary, this work supports the hypothesis of intra-cluster coordination and investigates the extent of this coordination.

\section{Background}

MicroRNAs (miRNAs) are small (22 nt) single-stranded non-coding RNA (ncRNA) molecules. They are processed from hairpin precursors of approximately $70 \mathrm{nt}$ (pre-miRNAs), which in turn are extracted from primary transcripts (pri-miRNA) [1,2]. miRNAs can repress gene expression post-transcriptionally by binding to the 3 ' untranslated regions (3' UTRs) of their target mRNAs [3-5]. In animal genomes, miRNAs are found in various genomic locations; while most are located in intergenic regions, some are found to be hosted within the introns of pre-mRNAs or within longer ncRNA genes [6,7]. Interestingly, known miRNA genes, both hosted and non-hosted, are often observed to be clustered [8]. A cluster usually includes two 
or three miRNA genes, but larger clusters have also been identified, including a human mir-17 cluster comprising 6 miRNA genes and a human mir-302 cluster comprising 8 miRNA genes. Baskerville and Bartel found that miRNAs within $50 \mathrm{~Kb}$ are highly correlated in expression across 24 different human organs, indicating that $50 \mathrm{~Kb}$ might be used as preliminary definition for miRNA cluster [9].

Although miRNAs in a given cluster are usually phylogenetically related in sequence, many human clusters containing miRNAs without apparent sequence homology are also found [10]. A plausible but yet-to-be validated possibility is that the clustered miRNAs are functionally related by virtue of targeting the same gene or different genes in the same pathway [8,10-12]. Studies of individual cases have to some extent supported this hypothesis. For instance, mir-15a-16 cluster act as tumor suppressor genes in prostate cancer by controlling cell survival, proliferation and invasion [13]. In gastric cancer, mir-106b cluster and mir-222 cluster are upregulated and modulate cell cycle by targeting the Cip/Kip family proteins [14]. The frequently studied mir-17-92 cluster acts not only in tumor formation but also in development of heart, lungs, and immune system [15-18]. Three individual miRNAs of the mir-379-410 cluster are required for activity-dependent development of hippocampal neurons [19]. mir-48 and mir-241 of a let-7 family cluster function together to regulate developmental timing in Caenorhabditis elegans [20]. However, apart from these individual cases, the hypothesis has not yet been systematically investigated.

Here, we conduct a comprehensive analysis of the conservation and functional consequence of miRNA clustering. We show that clustered miRNAs have functional relatedness through co-targeting proteins in close proximity of PPI network.

\section{Results}

\section{MiRNA clusters' construction and property}

It has been reported that clusters may contain unrelated miRNAs $[21,22]$. But the frequency of such heterogeneity has not been comprehensively evaluated. Prior research [10] has identified the clustering of 326 registered human miRNA genes, using the strategy proposed by Altuvia et al. [23]. According to miRBase [24], the number of registered miRNA genes has doubled. Still more homologs of the known miRNAs are expected in human genome, especially when more and more miRNA genes derived from TEs (transcriptional elements) have recently been identified [25-27].

In order to analyze the genomic clustering of miRNAs as systematically and exhaustively as possible, we conducted a cross-species Blast search of known pre-miRNA sequences in the entire human, mouse and rat genomes. In rat genome we found 152 novel miRNA loci that are homologues of previously annotated human or mouse miRNAs, whereas the corresponding numbers of loci in the mouse and human genomes are 33 and 19, respectively (see Additional File 1). We clustered miRNA genes within $50 \mathrm{~kb}$ of each other according to Bartel et al. [9]. After filtering with RNA polymerase II promoter analysis software and expression profiles (for details, see Material and Methods), a total of 104 miRNA clusters representing 67 unique miRNA combinations have been identified in human genome. Clusters composed of only hsa-mir-566 were not included, since hsa-mir-566 is thought to be derived from repeat sequence $A l u$, which is pervasive in the human genome [28]. Similarly, 91 clusters representing 64 unique miRNA combinations were found in mouse (mmu-mir-680 excluded), and 56 miRNA clusters, all unique, were identified in rat.

In recent releases of miRBase, similar miRNA precursors are assembled into miRNA families (miFams) based on computational analysis and manual inspection. miRNAs in a family have been shown to be phylogenetically related $[29,30]$. We further mapped the miRNAs in our data to their families according to miRBase's assignment. As results, 55 distinct miRNA clusters were identified at family level in human genome, along with 51 in mouse, and 42 in rat (see Additional File 2). After mapping to families, 31 clusters have been found to be conserved among the three species, which take up 56\% in human, $61 \%$ in mouse and $74 \%$ in rat (see Additional File 3).

Approximately half of the identified clusters are composed of multiple miRNA families and are denoted as miRNA hetero-cluster throughout this paper. On average, a hetero-cluster contains three miRNA families, and around half of the miRNA genes in a hetero-cluster do not belong to the main family (Table 1). Additionally, even miRNAs in the same family show sequence divergence and possibly target different proteins. This cluster heterogeneity is unlikely to merely derive from random mutation and retention. The fact that these clusters have been fixed in several modern animal genomes implies some evolutionary advantages of such a miRNA gene organiza-

Table I: Heterogeneity analysis of the miRNA clusters.

\begin{tabular}{llll}
\hline & human & mouse & rat \\
\hline HEC/TC & $53 / 104$ & $47 / 91$ & $26 / 56$ \\
\hline AFH & 2.5 & 3.1 & 3.3 \\
\hline PNM & 0.49 & 0.52 & 0.53
\end{tabular}

HEC - The number of hetero-clusters. TC - Total number of clusters. AFH - Average number of families in a hetero-cluster. PNM - Average percentage of miRNAs do not belong to the main family in each hetero-cluster. 
tion, which may provide an efficient internal mechanism for them to function in coordination $[8,12,31]$.

\section{Functional Coordination on PPI network}

Coordination among regulators from the transcriptional and miRNA regulatory layers has recently drawn great interests $[32,33]$. To reveal why non-homologous miRNAs are found in the same cluster, we analyzed their potential coordination in the context of PPI networks. Generally, the coordination of miRNAs in a cluster should be analyzed directly through justifying the relative proximity between their targets in the network. However, because of the incompleteness of credible PPI networks (e.g. Human Protein Reference Database, HPRD) and the low reliability of other integrated databases, we primarily chose to reverse the procedure to analyze whether proteins in close proximity incline to be regulated by miRNAs from the same cluster (henceforth referred to as "sc-miRNAs").

We first investigated directly interacting protein pairs. It has recently been reported that interacting protein pairs have a certain propensity to be regulated by the same miRNA [34]. There may exist a similar tendency that two interacting proteins are also regulated by sc-miRNAs. ScmiRNAs can fulfil the same function through a complementary approach - by assembling two non-related miRNA loci in the same transcriptional unit. Before analysis, we prepare the data as follows:

We used HPRD as the golden standard positive (GSP, interacting protein pairs) dataset, and the dataset generated by Rhodes et al. [35] according to protein subcellular localization as the golden standard negative (GSN, noninteracting protein pairs) dataset. Comparisons of various miRNA target prediction programs suggest that TargetScan could achieve both high sensitivity and specificity $[36,37]$. We therefore employed TargetScan predictions for clustered miRNAs. For targets of a miRNA family, common targets shared by all its miRNA members were used. The number of miRNA sites is $9.7 \pm 8.9$ per transcript for GSP, and $10.1 \pm 9.4$ for GSN. To specifically evaluate the coordination among different miRNA families in a cluster, we constructed a family-represented version of heteroclusters by mapping miRNAs in a cluster to their families, and then having redundancies removed (We will later denote these clusters as family-represented hetero-clusters and clusters before collapsing as original clusters). As a result, the 53 hetero-clusters have been collapsed to 37 family-represented hetero-clusters. To curb possible bias in the target prediction and isolate the effects of clustering, a control was generated by randomly reshuffling miRNAs (or families, when studying family-represented heteroclusters) among the clusters.
Two measures were employed to assess sc-miRNAs' coordination: one measure is the number of clusters that regulate the two proteins, which reflects the strength of coordination; the other is the percentage of protein pairs that are regulated by sc-miRNAs, which reflects the range of coordination. We calculated the prevalence of miRNAs' coordination on the GSP and GSN datasets for real clusters and 1,000 random cluster sets. Interacting proteins are found to have a significantly higher tendency to be coregulated by miRNAs from real than from randomly organized clusters (Figure 1). This tendency towards coordinated regulation was not observed for the GSN dataset, which justifies our study of sc-miRNAs' coordination in the context of PPI network. The large coordination measurements detected in random cluster set might be due to: 1) considerable noise exists in current protein-protein interaction data and miRNA target prediction; 2) when reshuffling 95 miRNAs families (contains redundancies, when a family appears in more than one cluster) in 37 hetero-clusters, there is a chance that two families in a real cluster are assigned to a random cluster again; meanwhile, miRNAs from the same family, which usually locate in one or two clusters, were dispersed into more clusters in randomization, and hence substantially increased the number of clusters they coincided.

Since the interacting pairs demonstrate stronger propensity to be regulated by sc-miRNAs than non-interacting pairs, we next asked whether there are some correlation between the propensity and the distance between two proteins in the network. The shortest paths were calculated between all the proteins in GSP dataset. We found a significant negative correlation between the number of clusters regulating the two proteins and the length of the shortest path between them in the PPI network (Spearman's rank correlation, correlation coefficient Rs $=-0.24$, $\mathrm{P}<2.2 \mathrm{e}-16)$. This result suggests that distance in the PPI network is an important factor with respect to the coordinated regulation from sc-miRNAs, and lend further support to our previous finding.

We have also noticed the great variance in the number of clusters regulating an interacting protein pair. Still $50 \%$ of the directly interacting protein pairs are not co-regulated by miRNAs from same cluster, and the numbers of clusters that do regulate them vary from 1 to 14 . Despite the noises and data incompleteness, directly interacting pairs are still likely to enjoy different levels of tendency. Therefore, we studied another factor, connectivity, that might influence this propensity.

Connectivity, the number of neighbours, is one of the most important properties of a protein node in a PPI network. In general, it quantitatively measures functional complexity and importance of a protein node in the PPI 

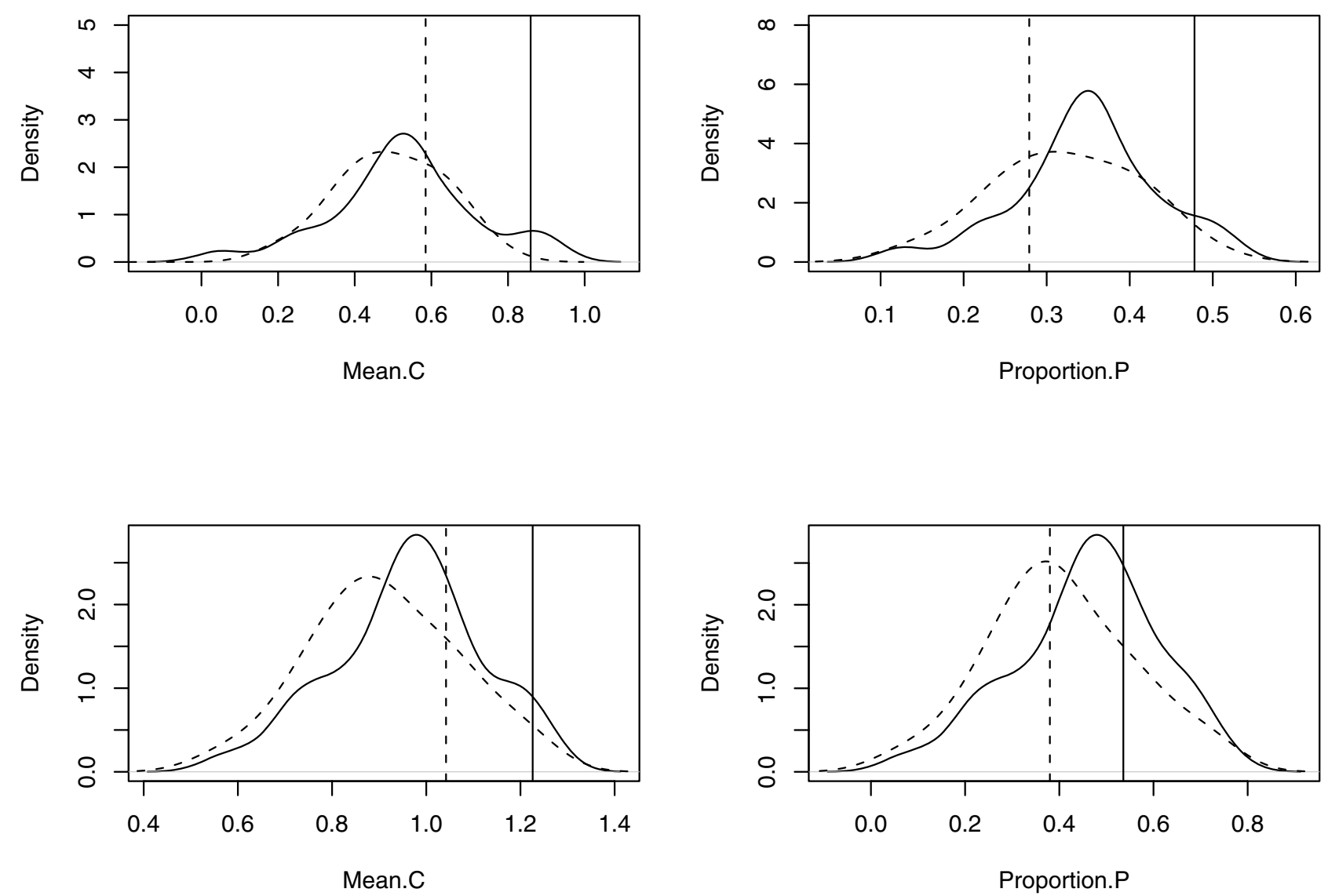

Figure I

The prevalence of coordinated regulation from sc-miRNAs. The left column shows the average number of clusters that co-regulate a protein pair (Mean.C) and the right column shows the proportion of protein pairs under co-regulation (Proportion.P). The first row shows Mean.C and Proportion.P calculated on the four combinations of GSP or GSN with family-represented hetero-clusters (HC) or its 1000 randomizations (RC, Random cluster set). GSP-HC is shown in solid line, GSN-HC in dashed line, GSP-RC in solid curve and GSN-RC in dashed curve. The second row shows Mean.C and Percent.P calculated on the four combinations of GSP or GSN with original clusters or its 1000 randomizations.

network. We counted all proteins' connectivity in HPRD network. The connectivity of proteins in GSP is $15.7 \pm$ 19.3, and in GSN $11.3 \pm 17.6$. We added up the connectivity of the two proteins in each pair and discovered a positive correlation between connectivity sum and the number of miRNA clusters targeting them in GSP (Spearman's rank correlation, correlation coefficient Rs $=0.11, \mathrm{P}$ $=3.136 \mathrm{e}-8$, as detailed in Table 2 ). More interestingly, this positive correlation is two times stronger in GSP than GSN (Rs is only 0.06 in GSN-HC, which is close to a random control). Considering the major difference between GSP and GSN is that the shortest path is longer than 3 between GSN proteins in HPRD network, this result indicates that the sc-miRNAs preferentially act on protein pairs within close proximity, rather than those with greater connectiv- ity (importance) in PPI network. It may explain why coordination has mainly been reported in regulating pathways.

To see whether these observations are robust, the same analysis was performed with PicTar prediction set, the overlap of TargetScan and PicTar prediction set as recommended by Sethupathy [36], as well as miRanda prediction set with alignment score greater than 155 (see Additional File 4). They all gave very consistent results.

\section{Modularity of the targets}

A module in networks is a local structure characterized by more internal than external links. Since our findings suggest that sc-miRNAs favour coordinated regulation of 
Table 2: A positive correlation between the sum of each two proteins' connectivity and the number of clusters regulating them.

\begin{tabular}{llllll}
\hline Spearman & & GSP-HC & GSN-HC & GSP-RC & GSN-RC \\
\hline Hetero-Clusters & Rs & 0.11 & 0.062 & 0.055 & 0.031 \\
\cline { 2 - 6 } & P & $3.136 \mathrm{e}-8$ & 0.002 & 0.006 & 0.217 \\
\hline Ori-Clusters & Rs & 0.118 & 0.058 & 0.035 & 0.045 \\
\cline { 2 - 6 } & P & $2.952 \mathrm{e}-9$ & 0.019 & 0.165 & 0.025 \\
\hline
\end{tabular}

Rs and P-value were from Spearman's rank test. For the test, 2500 pairs were randomly sampled from GSP and GSN respectively. The last two columns show the results calculated on a random cluster set.

local regions in PPI network, does sc-miRNAs' target gene set demonstrate some modularity? Xu and Wong [38] have found three miRNA clusters involved in regulating 15 signaling pathways by filtering mouse Biocarta pathway data. Pathway has been recognized as a common form of modularity in network analysis. Here, we explored another form of modularity, network community, in sc-miRNAs' targets with respect to HPRD network. Community structure in networks means the appearance of densely connected groups of vertices, with only sparser connections between groups [39]. We have found 32 network communities of size no less than 10 from HPRD network by joining cliques with size 3 to 7 together, using CFinder $2.0[40]$. We selected those network communities with at least $50 \%$ proteins targeted by a miRNA cluster and at most $40 \%$ regulated by any member of the cluster to guarantee that they are co-regulated by the cluster rather than any single member. For the 8 communitycluster pairs we obtained, the P-value was calculated based on the overlap of each community with the targets of one thousand simulated miRNA clusters of the same size. Finally, we obtained 5 community-cluster pairs with $\mathrm{P}<0.03$ (FDR $<0.2$, Table 3). Among them, mir-17 cluster's role in transcription regulation through this protein community is well-known $[41,42]$. Xie et al. recently reported mir-512 cluster's involvement in histone acetylation in embryonic stem cells [43]. Another interesting one is mir-379 cluster. It targets a network community of ten proteins that are involved in circadian rhythm (Figure 2). Although there is no experimental report on it, circadian rhythm is modulated by neural system while mir-379 cluster is brain-specific and is required for dendritogenesis [19].

Although our work mainly focus on protein-protein interaction data, for it is more complete and more suitable for global analysis, we re-ran our analysis with KEGG human pathway data, and presented the results in Additional File 4.

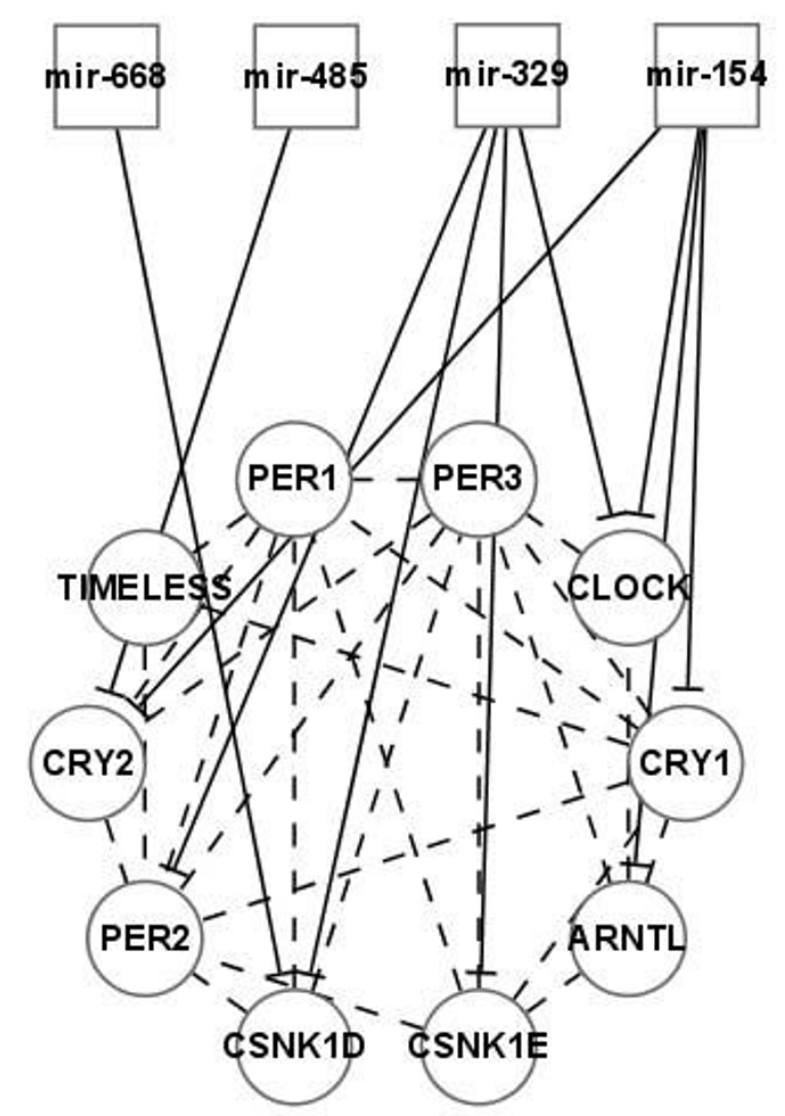

Figure 2

A network community of ten proteins is co-regulated by four miRNA families in mir-379 cluster. This network community is involved in circadian rhythm [KEGG pathway:hsa047l 0] and is co-regulated by nine miRNAs (mir-494, mir-543, mir-495, mir-38I, mir-539, mir-668, mir-485, mir-496, and mir-409) from four families in mir-379 cluster. We mapped these miRNAs to their families for the simplicity of illustration.

\section{Coordination and conservation}

Cluster provides a neat mechanism to transcribe many cooperative miRNAs simultaneously. It could hardly be imaged that this exquisite coordination has been generated in a single event; rather, it should have been consolidated through a long period of evolutionary process. To investigate the relationship between coordination and conservation, we have to calculate the target interactions between miRNAs in a cluster.

A comparison between human, mouse and rat miRNA clusters enables us to classify miRNAs in hetero-clusters into two categories: one is conserved miRNAs, which are observed in human, mouse and rat clusters; the other is non-conserved miRNAs, which exist in human clusters but are unobtainable in mouse or rat clusters. We counted the target interactions irredundantly within and between 
Table 3: Significant network communities that are enriched with target genes of miRNA clusters.

\begin{tabular}{llll}
\hline Chr & Cluster & Network Community & Function \\
\hline $3(-)$ & let-7 & $\begin{array}{l}\text { ACTAI, DRP2, SNTAI, DMD, DAGI, SNTB2, UTRN, SNTGI, SNTG2, KCNII2, } \\
\text { SNTBI, PGM5PI, DTNA, DTNB, DGKZ }\end{array}$ & muscle development and contraction \\
\hline $13(+)$ & mir-I7 & $\begin{array}{l}\text { AR, ESRI, RBI, TP53, CREBBP, EP300, JUN, STAT3, NCOAI, SMAD4, SMAD2, } \\
\text { SMAD3, BRCAI, STATI, TRIP4, RELA, CCNDI, SPI }\end{array}$ & positive regulation of transcription \\
\hline $14(+)$ & mir-379 & ARNTL, PERI, TIMELESS, CRYI, CLOCK, PER3, CRY2, PER2, CSNKID, CSNKIE & circadian rhythm \\
\hline $19(+)$ & mir-5I2 & HDAC2, RBBP7, RBPI, SAP30, HDACI, INGI, RBBP4, BRMSI, SIN3A, BRMSIL, & histone deacetylase \\
\hline $\mathrm{C}$ C2ORF59 & mir-450 & TP53, CUL5, COPS3, GPSI, COPS2, COPS8, COPS6, COPS5, COPS4, COPS7A & cancer suppressor p53 with COP9 signalosome \\
\hline
\end{tabular}

non-conserved miRNAs and conserved miRNAs. miRNA pairs with targets overlapping more than 100 within HPRD-recorded proteins were excluded because their mature sequences are so similar as to be suspected of having identical function. We calculated the empirical Pvalue of each miRNA pair's number of target interactions by 10,000 times randomization of miRNA-target protein association. The negative logarithm of P-value $(-\lg P)$ was then used to assess the coordination. The greater $-\lg P$ is, the stronger the coordination is. As results, $33.3 \%$ of conserved miRNAs pairs have been found to cooperate significantly in regulating PPI network $(-\lg P>1.5$, FDR $<0.1)$, which comports with our previous finding of intra-cluster coordination. Coordination within non-conserved miRNAs, however, is much weaker than that within conserved ones in a cluster (Figure 3, one-sided two-sample Kolmogorov-Smirnov test: $\mathrm{D}=0.34, \mathrm{P}=1.38 \mathrm{e}-11$ ). In other words, conserved miRNAs are generally more cooperative with each other than non-conserved ones in a cluster. The result is consistent on miRanda target set.

Although results in previous section indicate that miRNAs in a cluster may co-regulate proteins in close proximity of PPI network, they provide no information on each miRNA cluster's coordination. Here, those highly coordinated miRNA pairs $(-\lg P>1.5)$, conserved and non-conserved, come from 17 (30.1\%) hetero-clusters. We have found literature support for the function of 15 of them, all listed in Additional File 2. Thus it can be estimated that at least $30 \%$ of the miRNA hetero-clusters we can find evidence in current HPRD network for their intra-cluster coordination.

\section{Discussion}

Recently, global analysis of miRNA targets in the context of sets of genes or PPI network has drawn great interests [34,38,44-47]. It could also provide important insights into how miRNAs in a cluster cooperate with each other. In this work, we adopted a protein-centered perspective to start our analysis. We fixed a protein set and analyzed the relationship of miRNAs that target them. This perspective has advantages in avoiding the incompleteness of proteinprotein interaction data, and allowing us to analyze miRNAs' functionality according to the features of their targets.

By using the protein-centered perspective, we observed that directly interacting proteins incline to be regulated by miRNAs in the same cluster. The closer these proteins are in the PPI network, the more likely the targeting miRNAs are located in the same cluster. Proteins usually fulfill certain functions by means of interaction. If their functions

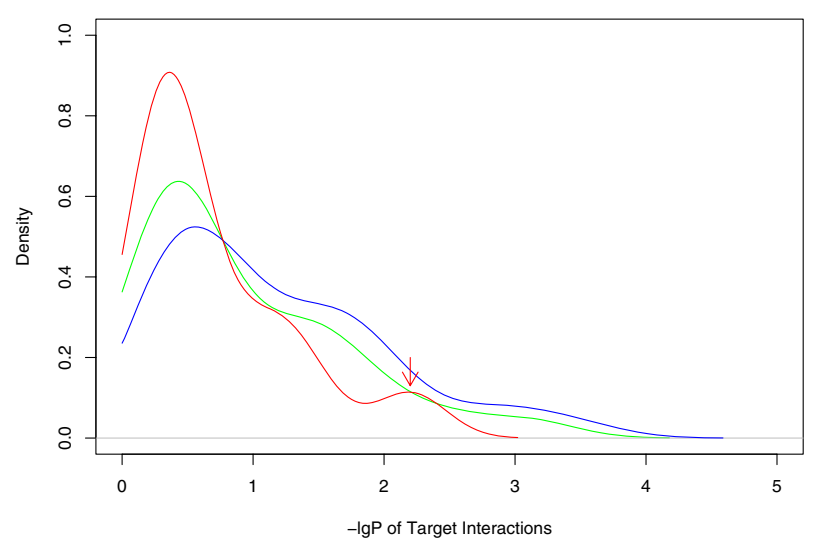

Figure 3

Coordination among non-conserved sc-miRNAs (red curve) is weaker than that among conserved sc-miRNAs (blue curve). - IgP of Target interactions between conserved and non-conserved sc-miRNAs is shown in green curve. The arrow-pointed peak in non-conserved miRNAs was formed due to coordination within rapidly evolving nonconserved families in mir-379 cluster. 

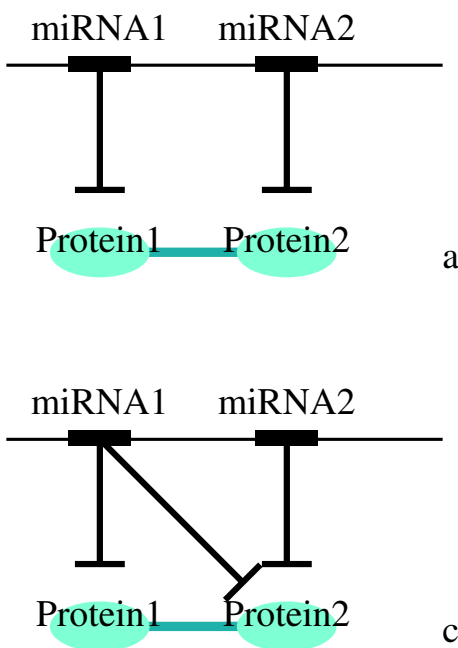

a.

c.

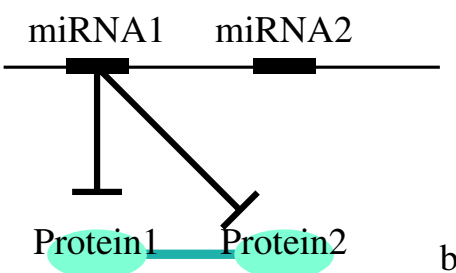

b.

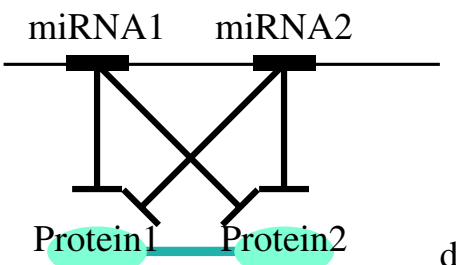

Figure 4

The four possible ways two proteins can be regulated by a miRNA cluster.

are temporarily not needed, then their expressions should be reduced simultaneously. miRNAs in a cluster transcribed as polycistron would provide a competent and efficient mechanism to achieve this goal. Our further analysis reveals that connectivity is another factor that matters only when proteins interact or are at least close in PPI network. Interacting proteins, both with great connectivity, usually connect two functional protein groups. They would be under stronger selective pressure to consent to a miRNA cluster's regulation. If one of them was not regulated in time, the module it involved would likely be still in function, and the coordination of the whole system would be greatly impaired.

The finding that distance is more important than connectivity suggests that sc-miRNAs preferentially co-regulate proteins in close proximity of a PPI network. However, this may also be due to the fact that PPI network are mainly gathered from high-throughput experiments which focus on physical interactions, such as yeast two hybrid system and tandem affinity purification. Therefore, for two proteins with long distance in PPI network, even their concurrence in time and space could not be guaranteed. To overcome this limitation, other information, such as signaling network or transcriptional regulatory network, would be needed [46,47].

\section{Conclusion}

This study supports the putative hypothesis of internal coordination among sc-miRNAs to regulate downstream biological networks. The linkage of sc-miRNAs' functional coordination and their targets' topological features we found highlights the potential to further investigate their subtle relationship.

\section{Methods \\ Data sources \\ Datasets of PPI}

Gold standard positive dataset: all the information in the Human Protein Reference Database (HPRD) has been manually extracted from the literature by expert biologists who read, interpret and analyze the published data. It contains 38,167 distinct interactions among 9,465 proteins [48]. Our analysis focused exclusively on the giant connected component of 9,134 proteins and 31,909 interactions. The remaining proteins were tiny clusters with sizes between two and eight. According to TargetScan's prediction, 8,264 of the 9,134 proteins are targeted by miRNAs. 6,737 of them are targeted by clustered miRNAs, and 4,835 are targeted by miRNAs that appear in heteroclusters.

Gold standard negative dataset: it was generated by Rhodes et al. [35], which includes all possible pair-wise combinations between two sets of proteins that are assigned a subcellular localization of the plasma membrane (1,397 proteins) and the nucleus $(2,224$ proteins), respectively, by the Gene Ontology (GO) Consortium. There are 2633 overlapping proteins between GSP and GSN.

\section{miRNA target prediction}

TargetScan's release 4.2 was downloaded from TargetScan site http://www.targetscan.org/. miRanda's prediction for 
418 human miRNAs in microRNA registry release 10.0 were downloaded from microRNA.org Jan 2008 release with align score higher than 155 . PicTar prediction was downloaded from UCSC genome browser PicTar miRNA track.

\section{Computational framework Determination of miRNA clusters}

We download all pre-miRNA sequences of Homo sapiens (hsa, 533 miRNAs), Mus musculus (mmu, 442 miRNAs) and Rattus norvegicus (rno, 285 miRNAs) from the miRNA registry release $10.0 \mathrm{http}$ //www.sanger.ac.uk/software/ Rfam/mirna/, and blast them against the whole genome of human, mouse and rat. Only the hits with 90\% coverage and 90\% identity were selected. All miRNA genes whose Blast distance is smaller than 100 bp were treated as one locus. According to Baskerville and Bartel [9], we considered loci within $50 \mathrm{~kb}$ of each other as belonging to the same cluster. miRNAs in a cluster were further screened using following filters:RNA polymerase II filter: Clusters were classified as intronic or intergenic according to their hostages. miRNA genes located at the introns or UTRs of protein-coding genes are transcribed together with their host genes, whereas miRNA genes dispersed in an intergenic region are generally believed to be transcribed by RNA polymerase II [49]. Therefore, we screened the regions surrounding intergenic miRNA clusters using the following RNA polymerase II promoter analysis software: Promoter Scan http://thr.cit.nih.gov/molbio/pros can for predicting promoter regions based on scoring homologies with putative eukaryotic Pol II promoter sequences, Promoter Prediction 2.0 http:// www.cbs.dtu.dk/services/Promoter/ for predicting transcription start sites of vertebrate Pol II promoters in DNA sequences, and NNPP (neural network promoter prediction) http://www.fruitfly.org/seq tools/promoter.html for predicting eukaryotic Pol II promoter sequences. Intronic miRNA clusters were directly reserved for transcription along with their host genes.

\section{Expression profile filter}

miRNAs in the clusters were also filtered according to expressional correlation. Three expression profiles [5053] were used in the screening. Only miRNAs with a Pearson Correlation Coefficient higher than 0.3 were retained in the cluster.

\section{Quantification of the functional coordination}

There are four possible ways two miRNAs in a cluster could target a protein pair (Figure 4). Situation b, concerning the propensity of two interacting proteins to be regulated by the same miRNA, regardless of miRNA clustering, has already be studied by Liang and Li [34]. In our analysis, we therefore excluded this situation. Situation c and d could be caused by the sequence homology of these two miRNAs, whose target sets hence greatly overlap, rather than true coordination. To eliminate the influence of sequence homology, we specifically measured the coordination among different miRNA families, where sequence homology is limited. We constructed a familyrepresented version of hetero-clusters by mapping miRNAs in a cluster to their families, and having redundancies removed. We separately performed the computation with family-represented hetero-clusters, and employed randomization as control.

\section{Randomized Control}

In our research, we have performed two types of randomization to construct control set: one is randomization of miRNA clusters. To obtain random controls for testing the propensity of interacting proteins to be regulated by scmiRNAs, we generated a cluster set by randomly shuffling miRNAs among clusters, while keeping the size of each cluster unchanged. The other is randomization of miRNAtarget protein associations. To obtain random controls for testing the proximity of sc-miRNAs' targets, we generated targets for each miRNA by randomly shuffling the miRNA-target protein associations, while keeping unchanged the number of proteins that a miRNA targets. The empirical P-value for target interactions was calculated against 10,000 independent randomized samples. The negative logarithm of P-value $(-\lg P)$ was then used to assess the coordination. The greater $-\lg P$ is, the stronger the coordination is.

\section{Others}

All the statistical tests and kernel density estimation were done in R. Connectivity and the shortest paths between each protein were calculated using iGraph package http:// cneurocvs.rmki.kfki.hu/igraph/.

\section{Authors' contributions}

$\mathrm{XY}, \mathrm{CL}$ and PY conceived of the study and carried out data analysis. YZ participated and supervised the study. SH participated in the identification of microRNA clusters. QL and SK participated in the study with useful suggestions. $\mathrm{XY}$ drafted the manuscript. All authors read and approved the final manuscript.

\section{Additional material}

\section{Additional file 1}

Novel miRNA loci in human, mouse and rat genomes. This files contains the novel miRNA loci we found in human, mouse and rat genomes. We found them through blasting all pre-miRNA sequences of the three species on their whole genomes. These novel loci were used later to identify miRNA clusters.

Click here for file

[http://www.biomedcentral.com/content/supplementary/17520509-3-65-S1.xls] 


\section{Additional file 2}

miRNA clusters found in human, mouse and rat genomes. This files contains two parts. Table $2 a$ contains the miRNA clusters we found in human, mouse and rat genomes and their positions. Table $2 b$ contains the miRNA clusters in which miRNAs have been mapped to their families according to miRBase's assignment. In this table 17 miRNA clusters, in which we have found significant coordination among miRNAs, are marked with red asterisk. Literature supports for the function of 15 of them are also listed.

Click here for file

[http://www.biomedcentral.com/content/supplementary/17520509-3-65-S2.xls]

\section{Additional file 3}

miRNA clusters' conservation among human, mouse and rat. This file contains miRNA clusters conserved or partially conserved among human, mouse and rat.

Click here for file

[http://www.biomedcentral.com/content/supplementary/17520509-3-65-S3.xls]

\section{Additional file 4}

Robustness on other data sets. This file contains the analysis performed on PicTar, TargetScan and PicTar overlapping, as well as miRanda predicted target set. It also contains significant KEGG human pathways that are enriched with target genes of miRNA clusters.

Click here for file

[http://www.biomedcentral.com/content/supplementary/17520509-3-65-S4.doc]

\section{Acknowledgements}

This work was supported by the National Sciences Foundation of China, under Grant Nos. 30570393 and 30600729 . The authors express special thanks to Dr. Dongbo Bu and Dr. Geir Skogerbo for editing and proofreading of this manuscript. The authors also thank anonymous reviewers for their valuable discussions and comments on this manuscript.

\section{References}

I. Lee Y, Jeon K, Lee JT, Kim S, Kim VN: MicroRNA maturation: stepwise processing and subcellular localization. Embo / 2002, 2I(I7):4663-4670.

2. Cullen BR: Transcription and processing of human microRNA precursors. Mol Cell 2004, 16(6):86I-865.

3. Lim LP, Glasner ME, Yekta S, Burge CB, Bartel DP: Vertebrate microRNA genes. Science 2003, 299(56 I 2): 1540

4. Ambros V: The functions of animal microRNAs. Nature 2004, 43 I (7006):350-355.

5. Bartel DP, Chen CZ: Micromanagers of gene expression: the potentially widespread influence of metazoan microRNAs. Nat Rev Genet 2004, 5(5):396-400.

6. Rodriguez A, Griffiths-Jones S, Ashurst JL, Bradley A: Identification of mammalian microRNA host genes and transcription units. Genome Res 2004, I4(I DA): 1902-1910.

7. Weber MJ: New human and mouse microRNA genes found by homology search. Febs J 2005, 272(1):59-73.

8. Kim VN, Nam JW: Genomics of microRNA. Trends Genet 2006, 22(3): $165-173$.

9. Baskerville S, Bartel DP: Microarray profiling of microRNAs reveals frequent coexpression with neighboring miRNAs and host genes. Rna 2005, I I (3):24I-247.

10. Yu J, Wang F, Yang GH, Wang FL, Ma YN, Du ZW, Zhang JW: Human microRNA clusters: genomic organization and expression profile in leukemia cell lines. Biochem Biophys Res Commun 2006, 349(I):59-68.
II. Bartel DP: MicroRNAs: genomics, biogenesis, mechanism, and function. Cell 2004, I I 6(2):28I-297.

12. Grun D, Wang YL, Langenberger D, Gunsalus KC, Rajewsky N: microRNA target predictions across seven Drosophila species and comparison to mammalian targets. PLoS Comput Biol 2005, I(I):el3.

13. Bonci D, Coppola V, Musumeci M, Addario A, Giuffrida R, Memeo L, D'Urso L, Pagliuca A, Biffoni M, Labbaye C, et al.: The miR-I 5a-miR16-I cluster controls prostate cancer by targeting multiple oncogenic activities. Nat Med 2008, I 4(I I): I 27 I-I 277.

14. Kim YK, Yu J, Han TS, Park SY, Namkoong B, Kim DH, Hur K, Yoo MW, Lee HJ, Yang HK, et al.: Functional links between clustered microRNAs: suppression of cell-cycle inhibitors by microRNA clusters in gastric cancer. Nucleic Acids Res 2009, 37(5): 1672-168I.

15. O'Donnell KA, Wentzel EA, Zeller KI, Dang CV, Mendell JT: c-Mycregulated microRNAs modulate E2FI expression. Nature 2005, 435(7043):839-843.

16. Ventura A, Young AG, Winslow MM, Lintault L, Meissner A, Erkeland SJ, Newman J, Bronson RT, Crowley D, Stone JR, et al.: Targeted deletion reveals essential and overlapping functions of the miR-17 through 92 family of miRNA clusters. Cell 2008, | 32(5):875-886

17. Xiao C, Srinivasan L, Calado DP, Patterson HC, Zhang B, Wang J, Henderson JM, Kutok JL, Rajewsky K: Lymphoproliferative disease and autoimmunity in mice with increased miR-17-92 expression in lymphocytes. Nat Immunol 2008, 9(4):405-4I4.

18. Lu Y, Thomson JM, Wong HY, Hammond SM, Hogan BL: Transgenic over-expression of the microRNA miR-17-92 cluster promotes proliferation and inhibits differentiation of lung epithelial progenitor cells. Dev Biol 2007, 3 I 0(2):442-453.

19. Fiore R, Khudayberdiev S, Christensen M, Siegel G, Flavell SW, Kim TK, Greenberg ME, Schratt G: Mef2-mediated transcription of the miR379-4 I 0 cluster regulates activity-dependent dendritogenesis by fine-tuning Pumilio2 protein levels. Embo J 2009, 28(6):697-710.

20. Abbott AL, Alvarez-Saavedra E, Miska EA, Lau NC, Bartel DP, Horvitz HR, Ambros V: The let-7 MicroRNA family members mir-48, mir-84, and mir-24I function together to regulate developmental timing in Caenorhabditis elegans. Dev Cell 2005, 9(3):403-4I4

21. Hertel J, Lindemeyer M, Missal K, Fried C, Tanzer A, Flamm C Hofacker IL, Stadler PF: The expansion of the metazoan microRNA repertoire. BMC Genomics 2006, 7:25.

22. Hayashita $Y$, Osada $H$, Tatematsu $Y$, Yamada $H$, Yanagisawa $K$, Tomida S, Yatabe Y, Kawahara K, Sekido Y, Takahashi T: A polycistronic microRNA cluster, miR-17-92, is overexpressed in human lung cancers and enhances cell proliferation. Cancer Res 2005, 65(2I):9628-9632.

23. Altuvia $Y$, Landgraf $P$, Lithwick $G$, Elefant $N$, Pfeffer $S$, Aravin $A$, Brownstein MJ, Tuschl T, Margalit $\mathrm{H}$ : Clustering and conservation patterns of human microRNAs. Nucleic Acids Res 2005, 33(8):2697-2706.

24. Griffiths-Jones S, Saini HK, van Dongen S, Enright AJ: miRBase: tools for microRNA genomics. Nucleic Acids Res 2008:D I54-I 58

25. Smalheiser NR, Torvik VI: Mammalian microRNAs derived from genomic repeats. Trends Genet 2005, 21(6):322-326.

26. Borchert GM, Lanier W, Davidson BL: RNA polymerase III transcribes human microRNAs. Nat Struct Mol Biol 2006, | 3(12): 1097-II01.

27. Piriyapongsa J, Marino-Ramirez L, Jordan IK: Origin and evolution of human microRNAs from transposable elements. Genetics 2007, I 76(2): 1323-1337.

28. Piriyapongsa J, Jordan IK: A family of human microRNA genes from miniature inverted-repeat transposable elements. PLoS ONE 2007, 2(2):e203.

29. Griffiths-Jones S, Grocock RJ, van Dongen S, Bateman A, Enright AJ miRBase: microRNA sequences, targets and gene nomenclature. Nucleic Acids Res 2006:D I 40-I44.

30. Huang Y, Gu X: A bootstrap based analysis pipeline for efficient classification of phylogenetically related animal miRNAs. BMC Genomics 2007, 8:66.

31. Li A, Mao L: Evolution of plant microRNA gene families. Cell Res 2007, 17(3):212-218. 
32. Shalgi R, Lieber D, Oren M, Pilpel Y: Global and local architecture of the mammalian microRNA-transcription factor regulatory network. PLoS Comput Biol 2007, 3(7): I I I.

33. Zhou Y, Ferguson J, Chang JT, Kluger Y: Inter- and intra-combinatorial regulation by transcription factors and microRNAs. BMC Genomics 2007, 8:396.

34. Liang $\mathrm{H}$, Li WH: MicroRNA regulation of human protein protein interaction network. Rna 2007, I3(9): I 402-I 408.

35. Rhodes DR, Tomlins SA, Varambally S, Mahavisno V, Barrette T, Kalyana-Sundaram S, Ghosh D, Pandey A, Chinnaiyan AM: Probabilistic model of the human protein-protein interaction network. Nat Biotechnol 2005, 23(8):95I-959.

36. Sethupathy P, Megraw M, Hatzigeorgiou AG: A guide through present computational approaches for the identification of mammalian microRNA targets. Nat Methods 2006, 3(II):88I-886.

37. Baek D, Villen J, Shin C, Camargo FD, Gygi SP, Bartel DP: The impact of microRNAs on protein output. Nature 2008, 455(7209):64-7I.

38. $\mathrm{Xu}$ J, Wong $\mathrm{C}$ : A computational screen for mouse signaling pathways targeted by microRNA clusters. Rna 2008 , 14(7): 1276-1283.

39. Newman ME, Girvan M: Finding and evaluating community structure in networks. Phys Rev E Stat Nonlin Soft Matter Phys 2004, 69(2 Pt 2):026II3.

40. Adamcsek B, Palla G, Farkas IJ, Derenyi I, Vicsek T: CFinder: locating cliques and overlapping modules in biological networks. Bioinformatics 2006, 22(8): $1021-1023$.

41. Petrocca F, Vecchione A, Croce CM: Emerging role of miR-106b25/miR- I 7-92 clusters in the control of transforming growth factor beta signaling. Cancer Res 2008, 68(20):819|-8194.

42. Yu Z, Wang C, Wang M, Li Z, Casimiro MC, Liu M, Wu K, Whittle J, Ju X, Hyslop T, et al.: A cyclin DI/microRNA I7/20 regulatory feedback loop in control of breast cancer cell proliferation. J Cell Biol 2008, 182(3):509-517.

43. Xie W, Song C, Young NL, Sperling AS, Xu F, Sridharan R, Conway AE, Garcia BA, Plath K, Clark AT, et al: Histone h3 lysine 56 acetylation is linked to the core transcriptional network in human embryonic stem cells. Mol Cell 2009, 33(4):417-427.

44. Hsu CW, Juan HF, Huang HC: Characterization of microRNAregulated protein-protein interaction network. Proteomics 2008, 8( I 0): 1975-1979.

45. Gusev Y, Schmittgen TD, Lerner M, Postier R, Brackett D: Computational analysis of biological functions and pathways collectively targeted by co-expressed microRNAs in cancer. $B M C$ Bioinformatics 2007, 8(Suppl 7):SI6.

46. Cui Q, Yu Z, Purisima EO, Wang E: Principles of microRNA regulation of a human cellular signaling network. Mol Syst Biol 2006, 2:46

47. Cui Q, Yu Z, Pan Y, Purisima EO, Wang E: MicroRNAs preferentially target the genes with high transcriptional regulation complexity. Biochem Biophys Res Commun 2007, 352(3):733-738.

48. Peri S, Navarro JD, Amanchy R, Kristiansen TZ, Jonnalagadda CK, Surendranath V, Niranjan V, Muthusamy B, Gandhi TK, Gronborg M, et al.: Development of human protein reference database as an initial platform for approaching systems biology in humans. Genome Res 2003, I3(I0):2363-2371.

49. Lee Y, Kim M, Han J, Yeom KH, Lee S, Baek SH, Kim VN: MicroRNA genes are transcribed by RNA polymerase II. Embo J 2004, 23(20):405I-4060.

50. Liang $Y$, Ridzon D, Wong L, Chen C: Characterization of microRNA expression profiles in normal human tissues. $B M C$ Genomics 2007, 8:166.

51. Lu J, Getz G, Miska EA, Alvarez-Saavedra E, Lamb J, Peck D, SweetCordero A, Ebert BL, Mak RH, Ferrando AA, et al: MicroRNA expression profiles classify human cancers. Nature 2005, 435(7043):834-838

52. Williams AE, Perry MM, Moschos SA, Lindsay MA: microRNA expression in the aging mouse lung. BMC Genomics 2007, 8: 172

53. Lee EJ, Baek M, Gusev Y, Brackett DJ, Nuovo GJ, Schmittgen TD: Systematic evaluation of microRNA processing patterns in tissues, cell lines, and tumors. Rna 2008, I 4(I):35-42.
Publish with Bio Med Central and every scientist can read your work free of charge

"BioMed Central will be the most significant development for disseminating the results of biomedical research in our lifetime. "

Sir Paul Nurse, Cancer Research UK

Your research papers will be:

- available free of charge to the entire biomedical community

- peer reviewed and published immediately upon acceptance

- cited in PubMed and archived on PubMed Central

- yours - you keep the copyright
BioMedcentral 